\title{
Funny Games - Ein Spiel ohne Grenzen
}

Nina SCHIMMeL

Der Titel des Films, der Gegenstand dieses Aufsatzes sein wird, verspricht Funny Games (1997), also »lustige Spiele«. In Anbetracht des Genres 〉Thriller〈, dem dieser Film üblicherweise zugeordnet wird, scheint vielleicht der Verdacht einer gewissen Deplaziertheit der Titulierung nicht unangebracht. Für gewöhnlich soll ein Thriller den Zuschauer Angst erleben lassen, da nicht in erster Linie die Aufklärung eines Verbrechens, sondern das Verbrechen selbst im Zentrum des Films steht. Um welche Art von Spielen, die auch noch lustig sind, kann es also gehen? Dieser Frage möchte ich hier auf den Grund gehen.

Der Regisseur Michael Haneke drehte zwei Versionen seines Films: Die erste Fassung von 1997 entstand mit deutschsprachigen Schauspielern in Österreich, die zweite verlagerte Haneke zehn Jahre später in die USA, wo er sein Projekt mit amerikanischen Schauspielern noch einmal für ein englischsprachiges Publikum realisierte. Die Handlung hat er dabei bis auf die Veränderung des Schauplatzes und einiger kleiner Details unverändert beibehalten.

Das Besondere an Funny Games ist, dass der Film durch die Überspitzung der klassischen Elemente eines Thrillers dem Zuschauer seine eigene Schaulust vor Augen führt und sich dabei selbst als Mittel der Manipulation entlarvt. Diese provokative >Vorführung` des Zuschauers durch den Film provozierte kontroverse Diskussionen - sowohl bei Kinozuschauern als auch bei Kritikern. Ehe ich zu den Ergebnissen meiner Filmanalyse komme, möchte ich zunächst erläutern, was unter einem >Spiel zu verstehen ist, welche Spielformen es gibt und in welchem Verhältnis diese zum Komischen stehen. Auf der Grundlage dieser Überlegungen werde ich anschließend auf Funny Games eingehen und versuchen, die Frage zu beantworten, was an und/oder in diesem Film als `Spiel< bezeichnet werden kann. Dabei werde ich drei Ebenen berücksichtigen: die Kinoebene, die Filmebene und die Zuschauerebene. 


\section{Zu FUNNY UND GAME}

\subsection{Game}

Nach Johan Huizinga ist das Spiel vor allem ein freies und freiwilliges Handeln, das im Grunde genommen überflüssig ist und jederzeit ausgesetzt werden kann. Das Spiel ist >nicht so gemeint<; es steht außerhalb des gewöhnlichen Lebens: »So wenigstens stellt sich uns das Spiel an sich und in erster Instanz betrachtet dar: als ein Intermezzo im täglichen Leben, als Betätigung in der Erholungszeit und zur Erholung« (Huizinga 1956: 16). Mittels seiner Spannungselemente, etwa der Ungewissheit und Chance, vermag das Spiel, den Spielenden in den Bann zu ziehen, wohl wissend, dass das, was er tut, eben >nur ein Spiel ist. Noch einmal Huizinga: »Die Ziele, denen es [das Spiel] dient, liegen selbst außerhalb des Bereichs des direkt materiellen Interesses oder der individuellen Befriedigung von Lebensnotwendigkeiten« (ebd.).

Jedes Spiel hat Spielregeln, die von allen Beteiligten akzeptiert und befolgt werden müssen. Es findet zudem stets innerhalb einer bestimmten räumlichzeitlichen Grenze statt und hat seinen Sinn in sich selbst. Seine wesentlichste Eigenschaft, so Huizinga, ist seine Wiederholbarkeit. Innerhalb seiner räumlichen Begrenzung, wie z.B. der Arena, des Tempels, der Bühne oder der Filmleinwand, herrscht die vom bzw. für das Spiel geschaffene Ordnung und bringt so eine zeitweilige, begrenzte Vollkommenheit in das sonst durch Verworrenheit gekennzeichnete Leben (vgl. ebd.: 17).

\subsection{Funny}

Das Lachen und das Komische sind, mit Huizinga gesprochen, ebenso wie das Spiel dem »Nichternst« (ebd.: 50) zuzusprechen. Dennoch bilden beide keine festen Bestandteile des Spiels: „An sich ist Spiel nicht komisch, weder für den Spieler, noch für den Zuschauer« (ebd.: 13). Und weiter: »Wenn wir eine Posse und ein Lustspiel komisch finden, geschieht es nicht wegen der Spielhandlung selbst, sondern wegen des Gedankeninhalts« (ebd.). Obwohl die Ernsthaftigkeit also kein Charakteristikum des Spielens ist, so birgt doch eine Vielzahl von Spielen ein Potenzial von Ernsthaftigkeit in sich. Der sportliche Wettkampf beispielsweise wird >im Ernst betrieben und erfüllt zugleich alle Kennzeichen eines Spiels (vgl. ebd.: 53f.). Auch bei Roger Caillois bildet der Wettkampf oder Agon eine Kategorie des Spiels - neben Zufall, Maskierung und Rausch - bei der sich die Rivalen in einer spezifischen Disziplin messen (vgl. 1982: 19). Kinder, aber auch Fußballspieler, nehmen ihr Spiel sehr ernst (vgl. Huizinga 1956: 13). Dies 
gilt auch für diejenigen, die gar nicht selbst an einem Spiel teilnehmen, aber dafür aus der Beobachtung desselben eine ernste Angelegenheit machen: »Der Ausgang eines Spiels oder eines Wettkampfes wird nur für die wichtig, die sich als Mitspieler oder Zuschauer [...] in die Sphäre des Spiels begeben und seine Regeln angenommen haben« (ebd.: 54).

Für das Wort »funny« im Titel des hier analysierten Films ließe sich zuletzt auch noch eine weitere Bedeutungsebene finden, nämlich in dem Sinn wie eine der Hauptfiguren den Begriff gebraucht: als Bezeichnung für ein »komisches« (Funny Games: 00:17:40) - im Sinne eines »seltsamen« - Spiel, das sich nicht als Spiel zu erkennen gibt.

\subsection{Funny Games}

Trotz der scheinbaren Gegensätzlichkeit zwischen dem kombinierten Begriffspaar »funny« und »game«, stellt sich der Titel Funny Games als treffende Pointierung heraus. Mit diesem Begriff werden in der Computerspiel-Terminologie alternativ auch Jump'n Run-Spiele bezeichnet, deren Hauptmerkmal die Geschicklichkeit ist. Sie zeichnen sich durch eine farbenfrohe Spielwelt aus, die auf der Tonebene von einer dazu passenden fröhlichen Musik unterstützt wird. Die Spieler sollen auf diese Weise »in eine entsprechende beschwingte Stimmung versetzt [werden] « (Eichner 2005: 480). Dabei ist die Spielfigur zumeist comicartig gestaltet. Der Spieler muss sie etwa durch Labyrinthe steuern oder sie hüpfen und springen lassen, um Aufgaben zu lösen und an das Ziel zu gelangen.

Vor dem Hintergrund dieser allgemeineren Anmerkungen möchte ich mich nun im Detail der deutschsprachigen Fassung von Funny Games widmen.

\section{Der Film Funny Games}

Die Frage, die sich stellt, ist nun: Liegt in Hanekes Film nach Huizingas Definition überhaupt ein Spiel vor - so wie der Titel es zu versprechen scheint? Um eine Antwort geben zu können, ist es notwendig, die verschiedenen Ebenen (Kino-, Film- und Zuschauerebene) zunächst einzeln näher zu beleuchten.

\subsection{Die Kinoebene}

Wenn man die oben genannten Grundprinzipien eines Spiels mit denen des Kinofilms vergleicht, so lässt sich zunächst festhalten, dass dieser zahlreiche Merkmale eines Spiels aufweist: Die Vorführung eines Kinofilms findet immer 
zu einer bestimmten Zeit und innerhalb einer bestimmten, in Bezug auf Dauer und Raum, begrenzten Situation statt. Die Zuschauer lassen sich als (passive) >Mitspieler auf die Regeln des Films bzw. die Bedingungen der Kinosituation ein: Sie akzeptieren etwa, dass es für dessen Dauer dunkel ist, dass nicht gesprochen werden darf und dass sie auf der Leinwand ein - zumeist - fiktionales Geschehen verfolgen. Während des Films weiß der Zuschauer, dass es sich >nur um einen Film $<$ handelt, und trotz dieser Gewissheit vermag das Mitverfolgen des fiktiven Geschehens Affekte wie Freude oder Angst bei ihm zu erzeugen. Auch bei einem Spiel wissen wir wie schon mit Huizinga beschrieben, dass es >nur ein Spiel ist, und dennoch lassen sich gewisse Emotionen, wie z.B. Wut, Freude oder Trauer nicht vermeiden. Darin erkennt auch Kendall Walton einen Zusammenhang zwischen einem Spiel und dem Rezipieren eines Films, den er als ein "game of make-believe (1976: 10) bezeichnet. Wie das Spiel sticht der Film aus dem wirklichen Leben heraus, als ein Freizeitvergnügen. Der Zuschauer (im Sinne eines >Mitspielenden`) kann sich der Kinosituation (als Spiel) immer wieder aussetzen oder aber - sollte es ihm nicht gefallen - den Kinosaal verlassen. Damit würde er gemäß dieser Logik die Spielsituation beenden.

Wenn man sagen kann, dass es sich beim Film und/oder bei einer Filmsituation um ein Spiel handelt, dann muss man - mit Huizinga gesprochen - feststellen, dass der Regisseur von Funny Games, Michael Haneke, gewissermaßen ein Falschspieler oder auch ein Spielverderber ist, und das gleich auf mehreren Ebenen.

Sein Film scheint zunächst im Einklang mit den Motiven und den Regeln eines klassischen Thrillers zu stehen: Eine glückliche Kleinfamilie (Vater Georg, Mutter Anna, Sohn Schorschi und Hund Rolfie) fährt mit ihrem Boot zum Haus am See. Diese Idylle und Ruhe wird durch zwei Eindringlinge ${ }^{1}$ ge- bzw. zerstört, indem sie erst höflich, dann mit Gewalt in das Haus eindringen. Es scheint allerdings, als würde der Film Funny Games die Regeln eines klassischen Thrillers nur deswegen einhalten, um mit ihnen brechen zu können. Gebrochen wird nämlich nicht nur mit den für einen Thriller typischen Regeln, sondern auch mit dem erwarteten Handlungsverlauf. Der erste >Regelbruch` findet nach 28 Minuten

1 Wie Stefan Höltgen richtig bemerkt, sind die beiden Männer im Grunde namenlos. Obwohl Haneke sie »Peter« und »Paul« nennt, wird schnell deutlich, dass diese Namen zu der von ihnen errichteten Fassade gehören und beliebig austauschbar sind. So rufen sie sich später »Beavis« und »Butthead« oder auch »Tom« und »Jerry«. Analog dazu sind auch die von ihnen hervorgebrachten Gründe für ihre Taten stereotyper Natur (Drogensucht, Scheidungskind, gelangweilter Jugendlicher), von denen wahrscheinlich kein einziger zutrifft (vgl. Höltgen 2010: 309). 
statt: Paul zwinkert dem Zuschauer ein erstes Mal zu. Dann spricht er ihn auch noch direkt an, wodurch die Spielsituation als eine solche entlarvt und die Fiktionalität des Films offengelegt wird ${ }^{2}$. Im Vorfeld hatte Haneke zunächst so getan, als würde er der Erwartung einer geschlossenen Fiktion im Spielfilms entsprechen - ganz gemäß Huizingas Charakterisierung eines Spielverderbers:

»Der Spieler, der sich den Regeln widersetzt oder sich ihnen entzieht, ist Spielverderber. Der Spielverderber ist ganz etwas anderes als der Falschspieler. Dieser stellt sich so, als spielte er das Spiel und erkennt dem Scheine nach den Zauberkreis des Spiels immer noch an. Ihm vergibt die Spielgemeinschaft seine Sünde leichter als dem Spielverderber, denn dieser zertrümmert ihre Welt selbst.« (Huizinga 1956: 18f.)

Haneke wäre demnach ein Spielverderber, der für 28 Minuten die Rolle des Falschspielers einnimmt.

Die `Spielregel`, dass die Zuschauer in der Dunkelheit des Kinosaals verborgene Beobachter sind, wird von der Figur Paul als eine eben solche entlarvt, indem er die vierte Wand der Leinwand durchbricht. Er macht darauf aufmerksam, dass der Zuschauer gerade einen Film sieht, und dass er dabei von der Leinwand aus beobachtet wird. Damit verdirbt er ihm das schlichte Genießen der ungebrochenen Fiktion. Denn, dies macht Christian Metz in Der imaginäre Signifikant deutlich: Die Lust am Kinoerlebnis entsteht normalerweise gerade durch die Gewissheit der Unsichtbarkeit - dadurch, dass der Beobachtende im Dunkel des Kinoraums verborgen bleibt (vgl. Metz 2000: 77). Im und durch die Medialität des Filmischen wird in Funny Games somit das Verhältnis zwischen >Sehen und Gesehen Werden`, zwischen dem Sichtbaren und dem Unsichtbaren, dem Verbergen und dem Zeigen thematisiert, oder, wenn man so will: Es wird die intentionale Struktur des filmischen Narrativs offengelegt. Haneke erleuchtet, metaphorisch gesagt, den Kinoraum; er macht im Gegensatz zu den klassischen Filmen die Kulisse und mit ihr denjenigen sichtbar, der schaut. Indem die Darsteller nicht nur den Zuschauer als Zuschauenden entlarven, sondern auch noch auf die Situation aufmerksam machen, dass es sich um einen Spielfilm handelt, verändert sich der Blickwinkel. Problematisiert wird das Verhältnis zwischen Zuschauer und Film. Anders ausgedrückt: Haneke versucht das Blickregime ${ }^{3}$, wel-

2 Gleichzeitig, das zeigt Alexander Darius Ornella, stellt sich bei den Zuschauern eine Art des »Mitgefangen-Seins« ein (vgl. 2008: 220).

3 Zum Thema Blickregime vgl. Lacan (1978). Eine Zusammenführung von Blickregime und Film wird von dessen Schüler Slavoj Žižek (2001) vorgenommen. Damit macht Žižek Lacans Theorien sowohl für die Populärkultur als auch für die Filmtheorie 
ches das Blickfeld von einer verborgenen Stelle aus lenkt, in den Vorstellungsbereich des Rezipienten zu rücken, da es »in die Sichtbarkeit höchstens als Phantasma ein[treten kann]« (Elsaesser/Hagener 2011: 130).

Noch ein ungeschriebenes Gesetz wird in Funny Games gebrochen: das Töten eines Kindes. Dieses scheint üblicherweise im Film nur dann ethisch annehmbar zu sein, wenn sich Kinder bereits in Vampire oder Zombies verwandelt haben und sich somit außerhalb der Gruppe besonders unschuldiger und schützenswerter menschlicher Wesen befinden, zu der sie normalerweise gehören (vgl. dazu Schimmel 2013). Alfred Hitchcock beging einen solchen Regelbruch in Sabotage (1936). Als er in diesem Film einen Jungen sterben ließ, der ein Paket mit einer Bombe darin transportierte, war das Entsetzen des Publikums so groß, dass Hitchcock dies später in seinem berühmten Interview mit François Truffaut bereute (vgl. Truffaut 1960: 118). Michael Haneke nun lässt Paul den Sohn Schorschi vor den Augen seiner Eltern töten. Die Art und Weise, wie dies inszeniert ist, macht genau auf den Aspekt aufmerksam, dass es sich um eine Zäsur handelt: Die Ermordung des Jungen findet außerhalb des Filmbildes statt. Aufgrund der Trennung von Bild- und Tonebene sieht der Zuschauer eine unwichtige Nebenhandlung, während er die Tötung des Jungen nur hört. Karl Ossenagg schreibt dazu: »Haneke hat das eigentliche Geschehen auf die Tonspur verlagert, in der Erkenntnis, dass das Ohr, im Gegensatz zum Auge, ereignishaft strukturiert ist, d.h. es provoziert das Betroffensein und Involviertsein in weit höherem Maße« (2008: 67). Gerade durch das Aussparen der Bilder wird zum einen ein Bruch in der Erwartungshaltung der Zuschauer erzeugt, durch welchen die Wirkung der Szene an Intensität gewinnt und zum anderen offenbart sie eine erschreckende Selbsterkenntnis: den Wunsch, den Gewaltakt zu sehen. Durch diese dem klassischen Hollywoodkino zuwider laufende Technik wird darauf aufmerksam gemacht, wie alltäglich die Erwartungen von Gewaltdarstellungen im Film geworden sind.

Es lässt sich zusammenfassend sagen, dass durch Hanekes Offenlegungen und Regelbrüche, die allesamt filmische Konventionen untergraben, die geregelte Welt des Spiels/Films zusammenbricht und ihre genuinen Eigenschaften verliert.

fruchtbar. Für einen Überblick über die filmtheoretische Verortung von Lacans Theoriemodellen vgl. Elsaesser/Hagener (2011), siehe auch zum aktuellen Stand der Forschung zum Blickregime Elia-Borer/Sieber/Tholen (2011). 


\subsection{Die Filmebene}

Das Spiel, welches Peter und Paul mit der Familie spielen wollen, basiert auf einer Wette: »Wir wetten, dass ihr in 12 Stunden alle drei kaputt seid « (Paul in Funny Games: 00:40:09). Nach Huizinga ist eine Wette durchaus ein Spiel, jedoch nur, wenn die Regeln von beiden Seiten akzeptiert werden. Roger Caillois betont, dass es bei einer Wette notwendig ist, dass »die Menschen aufhören können, wann es ihnen gefällt, [sie] müssen sagen können: Ich spiele nicht mehr« (Caillois 1982: 12f.). Diese Entscheidungsfreiheit liegt bei der Wette von Peter und Paul nicht vor. Die Schobers werden dazu gezwungen, die Regeln anzuerkennen, die sie auch nicht verändern können (vgl. Schacht 2008: 267). Als die Familie sich weigert, dieses Spiel mitzuspielen, sagt Peter nur: »Die Wette läuft noch. Die kann man nicht einseitig aufkünden!« (Funny Games: 01:31:20). Nach Huizinga und Caillois gibt es somit überhaupt keine Spielsituation. Die Einzigen, welche die Regeln des Spiels festlegen und so manipulieren können, wie es ihnen passt, sind die beiden Eindringlinge. Daher ist es auch nicht verwunderlich, dass in der berühmten Rückspulsequenz nur Paul nach dem Zurückspulen des Erschießens von Peter sein Verhalten ändern und vor Anna zum Gewehr greifen kann ${ }^{4}$. Peter und Paul beherrschen die Lage; die Familienmitglieder sind bloße Figuren auf einem Spielbrett. So wird die eingangs erwähnte doppelte Bedeutung des Wortes funny wieder aufgenommen: Für die Familie Schober ist es ein >seltsames`Spiel, da sie zum Spielen gezwungen wird und es sich somit für sie eher um ein >Pseudo-Spiel handelt. Für die beiden Männer hingegen ist das Spiel, durch ihre (All-)Machtposition bedingt, durchaus ein >lustiges<.

Neben der Wette gibt es noch weitere Spiele, die Paul spielen möchte. Etwa Das Kätzchen im Sack, was in diesem Falle bedeutet, dass der kleine Schorschi einen Kissenbezug über den Kopf gestülpt bekommt, damit er nicht mit ansehen muss, wie seine Mutter gezwungen wird, sich auszuziehen (»zur Wahrung des moralischen Anstandes« (Funny Games: 00:45:00), wie Paul sagt). Gleichzeitig ist dieser >Schutz` aber auch ein Instrument, um die Mutter dazu zu zwingen zu tun, was von ihr verlangt wird. An diesem `Spiel ist noch ein anderer Aspekt interessant: Die Redewendung »die Katze im Sack kaufen« bezeichnet normaler-

4 Leland Monk hingegen schreibt in seinem Aufsatz Hollywood Endgames, es sei seltsam, dass die Mutter Anna nicht einfach nach der Fernbedienung greift und bis an den Anfang des Spiels zurückspult (vgl. 2010: 425). Dagegen bleibt einzuwenden, dass Peter und Paul die Regeln festgelegt haben, so dass sie diese auch nach ihrem Belieben verändern können. Anna hingegen ist nur eine Spielfigur, die nach diesen Regeln handeln muss. 
weise, dass man etwas kauft, ohne es vorher zur prüfen. In Funny Games wird das Sprichwort umgedreht, denn hier soll, um es metaphorisch auszudrücken, die Katze den Käufer nicht sehen und nicht umgekehrt.

Die Abzählreime gehören ebenfalls zu den beliebten Spielen in diesem >Spiel-Film< der besonderen Art: Es wird derjenige ausgezählt, der als nächstes sterben soll. Nach einem Fehler wird Paul von seinem Spielgefährten zur Einhaltung der Spielregeln ermahnt: Er solle nicht denjenigen töten, der ausgezählt wird, sondern denjenigen, der beim Auszählen übrig bleibt. Dies führt dazu, dass der Junge Schorschi zuerst sterben muss. Die beiden Männer verbinden somit die naivsten und kindlichsten Spiele, wie die Abzählreime, mit dem höchsten Ernst, dem Tod. Aber es werden nicht nur Kinderspiele instrumentalisiert und dekontextualisiert, sondern auch >unschuldiges`Gerät wie das Golfequipment von Vater Georg wird zweckentfremdet: Der Golfschläger, mit dem Paul - wie er sagt - nur einmal einen Abschlag machen möchte, wird dazu verwendet, den Hund der Familie zu töten und kurze Zeit später das Bein des Vaters zu zertrümmern. Das sportliche Spielgerät wird zu einer Waffe und zu einem Repressionsinstrument umfunktioniert.

Für gewöhnlich harmlose Spiele werden in Funny Games zum Medium der Umsetzung der psychopathischen Absichten und Neigungen der beiden Täter. Durch dieses Aufeinanderprallen der Gegensätze (vgl. Sterneborg 2011: 67) werden die Spiele ihrer Unschuld beraubt und verwandeln sich in blutigen Ernst. Gleichzeitig wird dabei aber das Spiel nicht nur zu etwas Ernstem, sondern auch der Ernst zu etwas Spielerischem, zu etwas Harmlosem stilisiert.

\subsection{Die Zuschauerebene}

Der Zuschauer ist in Funny Games fast genauso machtlos wie die Schobers: So wie Peter und Paul mit der Familie spielen, spielt der Regisseur Haneke mit ihm, indem er mit bestehenden Regeln (eines Thrillers, eines Films) bricht und seine eigenen festlegt. Es entsteht also eine Situation des Spielzwangs auf beiden Ebenen, aus der allerdings der Zuschauer, anders als die Familie, die Möglichkeit hätte, zu entfliehen - was er allerdings so lange nicht tut wie er in der Kinosituation verbleibt.

An sich besteht nämlich allein zwischen dem Zuschauer und den beiden Männern Peter und Paul eine Spielsituation, die man als >wirklich` (d.h. gemäß der Definition Huizingas als harmlos) bezeichnen kann. >Wirklich (ist die Spielsituation insofern, als der Zuschauer durch das Verweilen im Kinosaal die Regeln des Spiels akzeptiert, die von den Eindringlingen aufgestellt und der Familie aufgezwungen werden. Er erkennt somit das Spiel als ein solches an. Auf die 
Frage von Anna, warum sie denn nicht sofort umgebracht würden, antwortet Peter nur: »Sie dürfen den Unterhaltungswert nicht vergessen. Wir würden doch alle um unseren Spaß gebracht!« (Funny Games: 00:50:44). Durch die Verwendung der Worte »wir alle« wird deutlich, dass Peter nicht nur sich selbst und seinen Freund Paul meint, sondern dass er hier auch den Zuschauer in das Geschehen (und seine Aussage) mit einschließt. Es wird davon ausgegangen, dass der Zuschauer, der sich einen Thriller ansieht, nicht nur weiß, sondern auch erwartet, dass Gewalt gezeigt wird, daher - so Peters Aussage - können sie als die Täter nicht einfach damit aufhören. Dies würde schließlich zu einem vorzeitigen Ende des Spiels und der >Unterhaltung « führen. Spätestens mit dem Satz »Wir wollen dem Publikum doch etwas bieten« (Funny Games: 01:29:36) wird explizit ausgesprochen, dass Peter und Paul dieses Spiel womöglich sogar nur für die $\mathrm{Zu}$ schauer spielen. Durch dieses Aufdecken der Zuschauerfunktion wird den zunächst und für gewöhnlich distanzierten Beobachtern ihre Rolle als Opfer und Mittäter bewusst gemacht.

\subsection{Zusammenfassung}

Für Peter und Paul ist ihr Tun ein Spiel, denn: Sie bestimmen die Regeln, legen fest, wann, wo und wie lange das Spiel dauert. Nicht zuletzt können sie es bei beliebig vielen Leuten wiederholen. In der letzten Sequenz des Films fragt Paul bei Nachbarn der Familie Schober nach Eiern. Damit wiederholt sich die Szene, in welcher der erste Kontakt zwischen den Tätern und ihren Opfern in Funny Games zustande kam: Paul fragt bei Anna nach Eiern. Während Peter und Paul also ganz klar (und immer weiter) spielen, handelt es sich für ihre jeweiligen >Spielpartner nicht um ein Spiel, denn sie werden zur Einhaltung der Spielregeln gezwungen. Der Zuschauer zu guter Letzt akzeptiert, solange er sich weiterhin im Kinosaal befindet, die Regeln von Peter und Paul. Solange er zuschaut, wird gespielt und nur so lange ist es auch ein Spiel.

\section{WER VERLIERT?}

Die Grenze zwischen Spiel und Ernst wird zu einem Spiel banalisiert, das am Ende von Funny Games noch einmal ganz explizit zum Thema wird, wenn nämlich die beiden Männer über den Unterschied zwischen Wirklichkeit und Fiktion diskutieren. Paul verweist darauf, dass die Fiktion, die man in einem Film sieht, ebenso wirklich sei wie die Wirklichkeit, da man beide ja wahrnehmen könne. Er spricht hier, diesmal indirekt, erneut den Zuschauer an. Wenn man davon 
ausgeht, dass der Kinofilm eine Art von Spiel ist, wie ich eingangs gezeigt habe, so lässt sich folgern, dass für Paul kein Unterschied zwischen 〉Spiel und >Wirklichkeit< bzw. >Ernstく besteht. Durch diese Aussage schließt sich nun der Kreis zu den Geschicklichkeitsspielen, die Funny Games genannt werden. Für Paul ist alles ein Spiel, ein eben solches Computerspiel, bei dem man jede Sequenz, die man nicht erfolgreich gemeistert hat, einfach wiederholen kann - so wie bei der Rückspul-Sequenz im Film:

„Für Paul ist die Realität längst verschwunden, sie kämpft höchstens noch ihre letzten Gefechte in Gestalt von Menschen, die an der Unterscheidung von Spiel und Leben, VideoGame und Alltag festhalten - aber die müssen im Auftrag einer perfekten Spielewelt beseitigt werden!« (Metelmann 2003: 141)

So laufen die beiden Männer wie programmiert durch ihre (Spiele-)Welt, optisch im Film hervorgehoben durch ihre weiße Bekleidung ${ }^{5}$, die sie durchaus comicartig erscheinen lässt. Der Zuschauer ist dabei derjenige, der nur scheinbar bestimmen kann, ob und wie lange er dem Spiel beiwohnen möchte. Er kann zwar den Kinosaal verlassen oder aber - zu Hause auf dem Sofa - den Film auf Video oder DVD zurückspulen bzw. ausschalten. Damit würde dann das `Spiek allerdings nicht zu Ende gespielt. Wenn der Zuschauer dagegen den Film bis zum Ende sieht, wird er nur dadurch belohnt, dass das letzte Filmbild einfriert und die roten Buchstaben »Funny Games« darüber eingeblendet werden (vgl. Abb. 1).

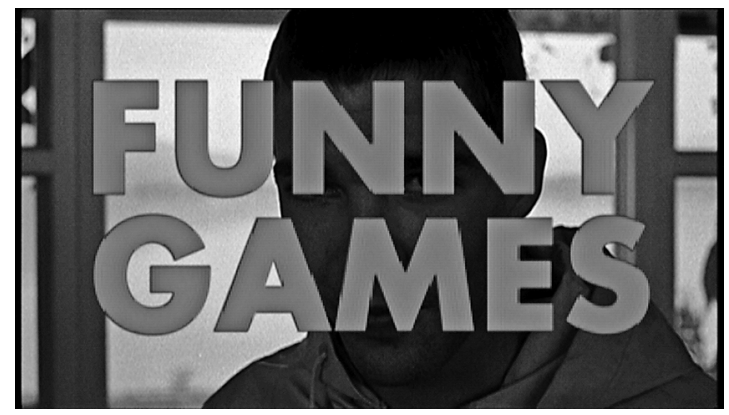

Abbildung 1: Letzte Einstellung des Films Funny Games

5 Die Bekleidung von Peter und Paul kann auch - und für diesen Hinweis danke ich Bettina Wodianka - als Zitat auf Stanley Kubricks A Clockwork Orange (1971) gelesen werden: Die ebenfalls weiße Kleidung von Alex und seinen Droogs fungiert dort als Maske, hinter der die wahren Absichten verborgen bleiben, und steht somit auch nur scheinbar für die Unschuld (vgl. dazu Sepp 2005: 245; Buovolo 2004). 
Dieser Effekt erinnert wiederum stark an das »Game Over« eines Computerspiels. Man könnte sich die Frage stellen, wer der Verlierer des Spiels ist. Meine Antwort darauf lautet: der Zuschauer. Ganz genau wie die Opfer im Film kann er nur verlieren - wenn er den Film ausschaltet oder wenn er ihn bis zum Ende sieht - in beiden Fällen verliert er. Weil, wie dem Opfer, auch dem Zuschauer selbst, nur die Illusion einer freien Entscheidung zur Verfügung gestellt wird.

\section{LITERATUR}

Buovolo, Marisa, »Masken der Gewalt. Die Sprache der Kleidung in A Clockwork Orange«, in: Kinematograph Nr. 19: Stanley Kubrick, Frankfurt a.M.: Deutsches Filmmuseum, 2004, S. 148-155.

Caillois, Roger, Die Spiele und die Menschen. Maske und Rausch, Frankfurt a.M.: Ullstein 1982.

Eichner, Susanne, »Videospielanalyse«, in: Lothar Mikos/Claudia Wegener (Hg.), Qualitative Medienforschung. Ein Handbuch, Konstanz: UVK 2005, S. 474-483.

Elia-Borer, Nadja/Sieber, Samuel/Tholen, Georg Christoph (Hg.), Blickregime und Dispositive audiovisueller Medien, Bielefeld: transcript 2011.

Elsaesser, Thomas/Hagener, Malte, Filmtheorie zur Einführung, Hamburg: Junius 2011.

Huizinga, Johan, Homo Ludens. Vom Ursprung der Kultur im Spiel, Hamburg: Rowohlt 1956.

Höltgen, Stefan, Schnittstellen. Serienmord im Film, Marburg: Schüren 2010.

Lacan, Jacques, Die vier Grundbegriffe der Psychoanalyse, Olten: Walter 1978.

Metelmann, Jörg, Zur Kritik der Kino-Gewalt. Die Filme von Michael Haneke, München: Fink 2003.

Metz, Christian, Der imaginäre Signifikant. Psychoanalyse und Kino, Münster: Nodus Publikationen 1992.

Monk, Leland, »Hollywood Endgames«, in: Roy Grundmann (Hg.), A Companion to Michael Haneke, Chichester: Wiley-Blackwell 2010, S. 420-438.

Ornella, Alexander Darius: »Das Spiel mit der Wirklichkeit. Gedanken zur medialen Konstruktion von Wirklichkeit anhand des Films FUNNY GAMES«, in: Christian Wessely/Gerhard Larcher/Franz Grabner (Hg.), Michael Haneke und seine Filme. Eine Pathologie der Konsumgesellschaft, Marburg: Schüren 2008, S. 213-221.

Ossenagg, Karl, »Der wahre Horror liegt im Blick. Michael Hanekes Ästhetik der Gewalt«, in: Christian Wessely/Gerhard Larcher/Franz Grabner (Hg.), 
Michael Haneke und seine Filme. Eine Pathologie der Konsumgesellschaft, Marburg: Schüren 2008, S. 53-82.

Schacht, Benjamin, »Rituale, Regeln und Paradoxien in Michael Hanekes Gesellschafts-Spielen «, in: Christian Wessely/Gerhard Larcher/Franz Grabner (Hg.), Michael Haneke und seine Filme. Eine Pathologie der Konsumgesellschaft, Marburg: Schüren 2008, S. 249-270.

Schimmel, Nina, »Untote töten? Zombiekinder im Film«, in: Christian F. Hoffstadt (Hg.), Tod. Aspekte der Medizinphilosophie, Bochum: Projekt-Verlag 2013 (im Erscheinen).

Seeßlen, Georg, Thriller. Kino der Angst, Marburg: Schüren 1995.

Sepp, Hans Rainer, »Die Maskierung der Gewalt. Stanley Kubricks A Clockwork

Orange«, in: Harun Maye/ders., Phänomenologie und Gewalt, Würzburg: Königshausen \& Neumann, 2005, S. 244-262.

Sterneborg, Anke, »Funny Games. Gewalt und Medien im Werk von Michael Haneke«, in: Thomas Koebner/Fabienne Liptay (Hg.), Film-Konzepte. Heft 21: Michael Haneke. München: edition text + kritik 2011, S. 65-75.

Truffaut, François, Hitchcock, London: Panther 1960.

Walton, Kendall, »Fearing Fictions«, in: The Journal of Philosophy 75, 1 (1978), S. 5-27.

Žižek, Slavoj, Die Furcht vor echten Tränen. Krystof Kieslowski und die »Nahtstelle«, Berlin: Volk \& Welt, 2001.

\section{FILME}

A Clockwork Orange (GB 1971, Regie: Stanley Kubrick).

Funny Games (AT 1997, Regie: Michael Haneke).

Sabotage (GB 1936, Regie: Alfred Hitchcock).

\section{ABBiLdUNGEN}

Abbildung 1: Filmstill aus dem Film Funny Games (1997). 